Konstanzer Online-Publikations-System (KOPS)

URL: http://www.ub.uni-konstanz.de/kops/volltexte/2008/4653/

URN: http://nbn-resolving.de/urn:nbn:de:bsz:352-opus-46532

First publ. in: The Journal of Physical Chemistry 87 (1983), 11, pp. 1873-1882

\title{
Investigation of Triplet Exciplex Dynamics by Magnetic Field Effects due to the Triplet Mechanism ${ }^{\dagger}$
}

\author{
T. Ulrich, U. E. Stelner," \\ Fakultät für Chemie, Universitat Konstanz, D-7750 Konstanz, Federal Republic of Germany
}

and R. E. Föll

Insttut für Physikalische Chemle, Universität Stuttgart, D-7000 Stuttgart-80, Federal Republic of Germany (Received: October 25, 1982; In Final Form: December 20, 1982)

\begin{abstract}
A reaction scheme is described allowing for magnetic field effects on the chemical kinetics of triplet reactions due to the selective decay of triplet sublevels. The theoretical treatment of this scheme is outlined on the basis of a stochastic Liouville equation, taking into account the rotational diffusion of molecules in liquid solution. Whereas the exact solution of the general case is obtained by a numerical procedure as described by Pedersen and Freed, an approximate analytical expression is also given. The magnetic field dependence of the radical yield in the electron transfer reaction between the thionine triplet and halogen-substituted anilines in methanol was measured up to $17 \mathrm{kG}$ by a microprocessor-controlled laser flash spectrometer with a special averaging technique. The data were analyzed in terms of the triplet mechanism operating on the decay of triplet exciplexes formed as intermediates in the electron transfer reaction. Whereas the rate constant of intersystem crossing $\left(k_{\text {isc }}\right)$ in these exciplexes depends on the halogen substituent and its position, the largest value $\left((7-10) \times 10^{10}\right.$ $\mathrm{s}^{-1}$ ) being obtained for the $p$-iodoaniline exciplex, the rate constant of exciplex dissociation into free radicals $\left(k_{\mathrm{fr}}\right)$ is of the order of $(5-8) \times 10^{9} \mathrm{~s}^{-1}$ for all halogen-substituted anilines investigated. Magnetic field effects on chemical kinetics according to the triplet mechanism appear to be especially useful for investigating very short-lived triplet intermediates not easily amenable to other methods of detection.
\end{abstract}

\section{Introduction}

Magnetic field effects in chemical kinetics are attracting more and more interest and advances in the field are progressing rapidly.1,2 The vast majority of effects ob-

\footnotetext{
${ }^{\dagger}$ Dedicated to Professor Albert Weller on occasion of his 60 th
} birthday. served during the past decade are explained in terms of the radical pair mechanism which originated from the

(1) Reviews have been given by P. W. Atkins and T. P. Lambert, Annu. Rep. Prog. Chem. A, 72, 67 (1975); A. L. Buchachenko, Russ. Chem. Rev. (Usp. Khim.), 45, 761 (1976); Y. N. Molin, R. Z. Sagdeev, and K. M. Salikhov, Sov. Sci. Rev., Sect. B, 1, 1 (1979). 
Scheme I

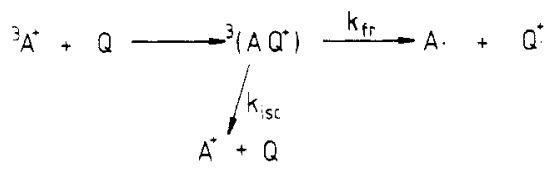

search for an understanding of CIDNP and CIDEP effects. $^{3}$ Although, however, it has been known for some time that there is an alternative mechanism for CIDEP effects, referred to as the triplet mechanism, ${ }^{4,5}$ no attention has been paid to the chemical kinetics aspect of this mechanism, except for some experiments at very low temperature ${ }^{6,7}$ where the triplet sublevels are kinetically isolated. Recently, we demonstrated the applicability of such a triplet mechanism to the decay kinetics of heavy atom substituted triplet exciplexes in room temperature liquid solutions. ${ }^{8}$

The triplet mechanism, as briefly characterized in the following, appears to provide a general principle for magnetic field modulation of photochemical reactions proceding via excited triplet states. In contrast to the CIDEP triplet mechanism where selective triplet sublevel population is essential, attention is focussed in our case on sublevel selective depopulation of a chemically reactive triplet. It can be shown that, unless spin relaxation among the triplet sublevels is much faster than the selective deactivation processes, the overall depopulation kinetics of the triplet is magnetic field dependent and so is the chemical product yield. In room temperature liquid solutions, the dominant relaxation mechanism establishing the sublevel equilibrium is molecular rotational diffusion, the time constant of which is usually much larger than the rate constants for radiationless decay or chemical reaction of excited triplets. This is probably the reason why effects of this kind have not been described earlier. Systematic investigations, however, with suitably selected systems should reveal magnetic field effects of this kind for a variety of different triplet reactions.

The triplet exciplexes considered in this paper have been previously postulated ${ }^{9}$ to be formed as intermediates (cf. Scheme I) in the quenching of the thionine triplet (electron acceptor ${ }^{3} \mathrm{~A}^{+}$) by aniline and its halogen derivatives (electron-donating quencher $\mathrm{Q}$ ), in order to properly account for the marked positional dependence of the heavy atom effect on the radical yield in this quenching reaction.

$\mathrm{By}$ a theoretical analysis ${ }^{8 \mathrm{~b}}$ of the spin-orbit coupling properties of the triplet exciplex ${ }^{3}\left(\mathrm{AQ}^{+}\right)$, it can be shown that heavy atom enhanced intersystem crossing (isc) deactivating the triplet exciplex to the ground state of its components must be sublevel selective. In particular, the triplet substate with the electron spin oriented parallel to the aromatic plane of the halogen-substituted aniline component of the exciplex should be least sensitive to the heavy atom effect on the isc rate. Thus, while the triplet

(2) A list of more recent work can be found in $\mathrm{Y}$. Tanimoto and $\mathrm{M}$. Itoh, Chem. Phys. Lett., 83, 626 (1981), ref 1-9.

(3) Cf. the reviews by A. Lepley and G. L. Closs, Ed., "Chemically Induced Magnetic Polarization", Wiley, New York, 1973; L. T. Muus, P. W. Atkins, K. A. McLauchlan, and J. B. Pedersen, Ed., "Chemically Induced Magnetic Polarization", Proceedings of the NATO Advanced Study Institute, Sogesto Urbino, Italy, Reidel, Dordrecht, 1977.

(4) P. W. Atkins and G. T. Evans, Mol. Phys,, 27, 1633 (1974).

(5) S. K. Wong, D. A. Hutchinson, and J. K. S. Wan, J. Chem. Phys., 58,985 (1973).

(6) M. Leung and M. A. El-Sayed, J. Am. Chem. Soc., 97, 670 (1975).

(7) R. H. Clarke, R. E. Connors, and J. Keegan, J. Chem. Phys. 66, 358 (1977).

(8) (a) U. Steiner, Chem. Phys. Lett., 74, 108 (1980); (b) Ber. Bunsenges. Phys. Chem. 85, 228 (1981).

(9) U. Steiner and G. Winter, Chem. Phys. Lett., 55, 364 (1978).
Scheme II

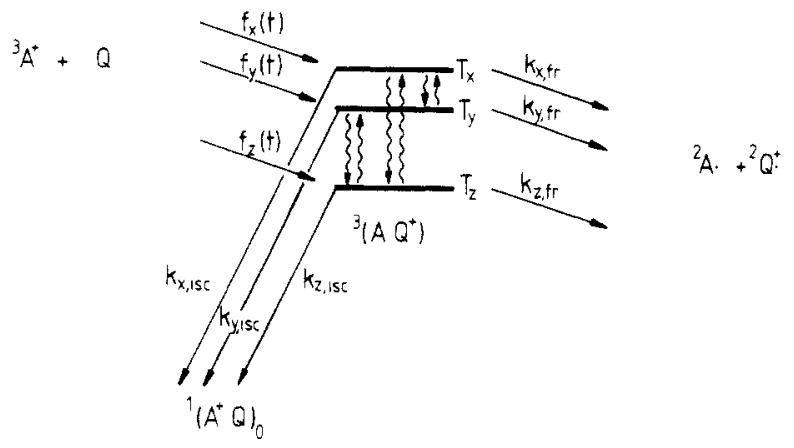

sublevels of the exciplex are probably populated at equal rates in the reaction of ${ }^{3} \mathrm{~A}^{+}$with $\mathrm{Q}$, one of them is expected to undergo much slower isc to the singlet ground state than the others. On the other hand, since dissociation of the triplet exciplex into free radicals (rate constant $k_{\mathrm{fr}}$ ), which is spin conserving, does not favor any one triplet substate, the triplet exciplex substate with the weakest isc should give the greatest contribution to the radical yield. Mixing of the slowly decaying substate with other ones by an external magnetic field will enhance its isc rate and reduce the radical yield.

From a quantitative investigation of this magnetic field effect it should be possible to extract the kinetic parameters of the triplet exciplexes in absolute units. Our previous theoretical treatment ${ }^{8}$ of the magnetic field modulation of quantum yields by the triplet mechanism made use of a rather intuitive approximate solution of the stochastic Liouville equation (SLE), which can be set up to give a physically consistent description of the mechanism. Although the principal conclusions from this approximate treatment are certainly correct, there remains some doubt as to the reliability of the numerical values of the parameters extracted from a comparison of theoretical and experimental results. On the other hand, the experimental accuracy available so far in our investigations did not justify a more elaborate analysis of the data. Meanwhile, we have arrived at an exact solution of the SLE ${ }^{10}$ have also greatly improved the experimental accuracy by use of a computer-controlled laser flash spectrometer, and have extended the range of applied fields from 4 to $17 \mathrm{kG}$. Making use of both theoretical and experimental improvements we demonstrate in this paper the potential of magnetic field studies for revealing the details of the dynamics of very short-lived triplet exciplexes.

\section{Theory}

A fairly general description of the kinetic situation to be considered is given in Scheme II. The substates $\mathrm{T}_{x}$, $\mathrm{T}_{y}$, and $\mathrm{T}_{z}$ of the triplet exciplex ${ }^{3}\left(\mathrm{AQ}^{+}\right)$are represented by distinct levels. The population kinetics is characterized by the functions $f_{i}(t)(i=x, y, z)$, whereby the population process corresponds to the reaction of the acceptor triplet ${ }^{3} \mathrm{~A}^{+}$with the electron donor $\mathrm{Q}$. Physical deactivation of the triplet exciplex occurs by isc to the singlet ground state with the corresponding rate constants, $k_{i, \text { isc }}$. Chemical quenching occurs via dissociation into the free radicals with rate constants $k_{i, \text { fr. }}$. Furthermore the triplet substates are kinetically connected by stochastic processes inducing the spin relaxation and by coherent processes (Larmor precession of the spin in an external magnetic field). As an analytical equivalent to the mechanistic Scheme II we use the SLE eq 1, which has been used in similar form by

(10) U. Steiner, to be published. 


$$
\begin{array}{r}
\dot{\rho}(\Omega, t)=i\left[\rho(\Omega, t), \mathbf{H}_{\mathrm{Z}}+\mathbf{H}_{\mathrm{D}}(\Omega)\right]_{-}+\Gamma_{\Omega} \rho(\Omega, t)+ \\
1 / 2[\rho(\Omega, t), \mathbf{K}(\Omega)]_{+}+\mathbf{W}(\Omega, t)
\end{array}
$$

Pedersen and Freed, ${ }^{11}$ in order to treat the triplet mechanism of CIDEP. The quantity $\rho(\Omega, t)$ is the three-dimensional spin density matrix of the triplet exciplex and $\Omega$ represents the set of Eulerian angles $\alpha, \beta, \gamma$ identifying the orientation of the exciplex. The commutator on the right-hand side of eq 1 describes the spin motion due to the Zeeman Hamiltonian $\mathbf{H}_{\mathbf{Z}}$ and the zero-field spin Hamiltonian $H_{D}(\Omega)$ given by eq 2 , where the $P_{i}(\Omega)$ are

$$
\mathbf{H}_{\mathrm{D}}(\Omega)=\sum_{i} E_{i} \mathbf{P}_{i}(\Omega)
$$

projection operators onto the molecular spin eigenstates $\mathrm{T}_{x}, \mathrm{~T}_{y}$, and $\mathrm{T}_{z}$ of the triplet exciplex, and the $E_{i}$ are their respective spin-spin coupling energies.

The second term on the right-hand side of eq 1 describes the stochastic motion of the molecular orientation, viz. rotational diffusion, the operator $\Gamma_{\Omega}$ being specified below.

The third term, [ ] denoting the anticommutator, has not been used as a sublevel-selective term in the treatments of the CIDEP triplet mechanism, $4,11,30$ though it has been noted by McLauchlan ${ }^{12}$ that sublevel-selective triplet depopulation could be a source of electron spin polarization. For the present problem the third term is of essential importance. It describes the combined chemical and physical decay of the triplet exciplex sublevels. The operator $\mathbf{K}(\Omega)$ is given by eq 3 .

$$
\mathbf{K}(\Omega)=-\sum_{i}\left(k_{i, \mathrm{isc}}+k_{i, \mathrm{fr}}\right) \mathbf{P}_{i}(\Omega)
$$

The last term $\mathbf{W}(\Omega, t)$ in eq 1 is the source term describing the triplet exciplex formation

$$
\mathbf{W}(\Omega, t)=\sum_{i} f_{i}(t) \mathbf{P}_{i}(\Omega)
$$

In using eq 1 as a basis of our treatment of the triplet exciplex magnetic field, we have, for simplicity, assumed that the tensor operators $\mathbf{H}_{D}$ and $\mathbf{K}$ have the same principal axes and that the dominant spin relaxation mechanism is provided by the stochastic modulation of the spin Hamiltonian due to rotational diffusion, an assumption which is also implicit to the current treatments of the CIDEP triplet mechanism. ${ }^{4,11}$ The numerical solution of eq 1 is described in detail elsewhere. ${ }^{10}$ It follows the method of Pedersen and Freed, ${ }^{11}$ which makes use of an orthogonal expansion of $\rho(\Omega, t)$ in the basis of the Wigner rotation matrices. To obtain the numerical results presented in the Discussion, we imposed a number of restrictions on the parameters introduced by Scheme II:

(1) In our experiments the triplet lifetimes of ${ }^{3} \mathrm{~A}^{+}$under conditions of quenching by $\mathrm{Q}$ was typically $1 \mu \mathrm{s}$, which is much longer than the spin relaxation time in room temperature liquid solutions of low viscosity. Since the triplet exciplex formation from ${ }^{3} \mathrm{~A}^{+}$is spin conserving, it is not likely that there is spin selectivity introduced by the exciplex population process. Therefore $\mathbf{W}(\Omega, t)$ is independent of $\Omega$. Furthermore, since we are interested here in the radical yield after complete triplet decay, the explicit time dependence of $\mathbf{W}(t)$ is not of interest, so that the source term can be accounted for by a simple $\delta$-function time dependence

$$
\mathbf{W}(t)=\frac{1}{24 \pi^{2}} \delta(t) \mathbf{I}
$$

(11) J. B. Pedersen and J. H. Freed, J. Chem. Phys., 62, 1706 (1974) (12) K. A. McLauchlan in "Chemically Induced Magnetic Polarization" L. T. Muus, P. W. Atkins, K. A. McLauchlan, and J. B. Pedersen, Ed., Proceedings of the NATO Advanced Study Institute, Sogesto Urbino, Italy, Reidel, Dordrecht, 1977, pp 151-167. where $I$ is the unit matrix.

(2) With respect to $\mathbf{H}_{D}$ and $\mathbf{K}$, the exciplex is treated as axially symmetric, i.e.

$$
\begin{gathered}
E_{x}=E_{y}=D / 3 \\
E_{z}=-2 / 3 D \\
k_{x, \text { isc }}=k_{y, \text { isc }}=k_{\text {isc }} \\
k_{z, \text { isc }}=z k_{\mathrm{isc}}
\end{gathered}
$$

where

$$
0 \leq z \leq 1
$$

In our previous analysis ${ }^{8}$ of spin-orbit coupling in halogen-substituted triplet exciplexes, it was shown that in zero order there is no heavy atom effect on spin-orbit coupling between $T_{z}$ and $S_{0}$ if the exciplex possesses an ideal sandwich configuration. Due to vibrational motions, however, which tilt the aromatic planes of the exciplex with respect to each other, the condition $k_{z, \text { isc }}=0$ should be relaxed.

(3) For the $k_{i, f r}$ it is assumed that they are not sublevel selective, i.e.

$$
k_{x, \mathrm{fz}}=k_{y, \mathrm{fr}}=k_{z, \mathrm{fr}}=k_{\mathrm{fr}}
$$

since spin will probably be conserved during exciplex dissociation.

(4) The rotational diffusion of the triplet exciplex is treated as isotropic, eq 10 , with $D_{\mathrm{r}}$ as the rotational dif-

$$
\Gamma_{\Omega}=D_{\mathrm{r}} \nabla^{2}
$$

fusion constant and $\nabla^{2}$ as the rotational diffusion operator. ${ }^{11}$

With restrictions (1)-(4), we obtain from the solution of eq 1 the yield of free radicals $\phi_{\mathrm{fr}}$ as a function of the following parameters:

$$
\phi_{\mathrm{fr}}=\phi_{\mathrm{fr}}\left(k_{\mathrm{fr}}, k_{\mathrm{isc}}, z, D_{\mathrm{s}}, D, \omega\right)
$$

where $\omega$ is the electronic Larmor frequency, given by

$$
\omega=\mu_{\mathrm{B}} g B / \hbar
$$

with $\mu_{\mathrm{B}}$ the Bohr magneton, $g$ the electronic gyromagnetic ratio, $B$ the magnetic flux density, and $\hbar$ the Planck constant.

Zero-Field Case. Whereas an exact closed form expression of the function $\phi_{\mathrm{fr}}$ cannot be given for the general case, this is possible for the zero-field case. For $z=0$ the correct expression has already been given in ref 8 . For $z$ $\neq 0$ it is readily generalized with the result

$\begin{aligned} \phi_{\mathrm{fr}}= & k_{\mathrm{fr}}\left(k_{\mathrm{fr}}+1 / 3(1+2 z) k_{\mathrm{isc}}+6 D_{\mathrm{r}}\right) /\left[\left(k_{\mathrm{fr}}+k_{\mathrm{igc}}\right) \times\right. \\ & \left.\left(k_{\mathrm{fr}}+4 D_{\mathrm{r}}\right)+2 k_{\mathrm{fr}} D_{\mathrm{r}}+z k_{\mathrm{isc}}\left(k_{\mathrm{fr}}+k_{\mathrm{igc}}+2 D_{\mathrm{r}}\right)\right]\end{aligned}$

Approximation for Intermediate Fields. An expression analogous to eq 13 can be used as an approximation for $\phi_{\mathrm{fr}}$ in the presence of a magnetic field if the zero-field splitting parameter $D$ is negligible. The magnetic field influence can be accounted for by substituting for $D_{\mathrm{r}}$ an effective rotational diffusion constant $D_{\mathrm{r}, \mathrm{B}}$ given by

$$
D_{\mathrm{r}, \mathrm{B}}=\frac{s\left(1-s p_{s}\right)}{6 s p_{s}-2}
$$

where $p_{s}$ is the Laplace transform of the spin correlation function $p\left(T_{i}, t ; T_{i}, 0\right)$ giving the probability of finding the molecule in substate $T_{i}$ at time $t$, if it was in the same substate at $t=0$ and if there are no overall triplet decay 
processes. This correlation function was derived in ref 8 . The Laplace transform is

$$
\begin{aligned}
& p_{\mathrm{s}}=\frac{1}{15}\left(\frac{5}{s}+\frac{2}{s+6 D_{\mathrm{r}}}+\frac{4\left(s+6 D_{\mathrm{r}}\right)}{\left(s+6 D_{\mathrm{r}}\right)^{2}+\omega^{2}}+\right. \\
& \left.\frac{4\left(s+6 D_{\mathrm{r}}\right)}{\left(s+6 D_{\mathrm{r}}\right)^{2}+4 \omega^{2}}\right)
\end{aligned}
$$

The best approximation to the exact $\phi_{\mathrm{fr}}$ is obtained by letting

$$
s=k_{\mathrm{fr}}+2 / 3 k_{\mathrm{isc}}
$$

which is just the average decay constant of the triplet exciplex. The expression derived previously ${ }^{8}$ corresponds to using eq 13-15 with $s \rightarrow 0$. It gives acceptable agreement with the exact results only if the rotational diffusion is not much slower than the triplet decay processes. Use of eq 16 , however, leads to generally good results for all values of the parameters only if $D$ is negligible.

High-Field Case. In the high-field case the exact treatment of eq 1 is considerably simplified. The eigenstates of the Zeeman Hamiltonian can be treated as decaying independently of each other and the dipolar spin-spin coupling operator $\mathbf{H}_{D}$ can be neglected. Let $\boldsymbol{p}_{\|}$ be the probability that the exciplex is in the states $T_{1}$ or $\mathrm{T}_{-1}$ and $p_{\perp}$ the corresponding probability for $\mathrm{T}_{0}$. Then, with $\beta$ the polar angle between the magnetic field direction and the molecular $z$ axis, one obtains

$$
\begin{gathered}
\dot{p}_{\|}(\beta, t)= \\
{\left[D_{\mathrm{r}} \nabla_{\beta}^{2}-k_{\mathrm{fr}}-1 / 2(1+z) k_{\mathrm{isc}}-1 / 2 \cos ^{2} \beta(1-z) k_{\mathrm{isc}}\right] p_{\|}(\beta, t)} \\
\dot{p}_{\perp}(\beta, t)=\left[\mathrm{D}_{\mathrm{r}} \Delta_{\beta}^{2}-k_{\mathrm{fr}}-k_{\mathrm{isc}}+\cos ^{2} \beta(1-z) k_{\mathrm{igc}}\right] p_{\perp}(\beta, t) \\
\nabla_{\beta}^{2}=\frac{\partial^{2}}{\partial \beta^{2}}+\cot \beta \frac{\partial}{\partial \beta}
\end{gathered}
$$

From $p_{\|}(\beta, t)$ and $p_{\perp}(\beta, t)$ the radical yield is obtained according to

$$
\begin{gathered}
\phi_{\mathrm{fr}}=k_{\mathrm{fr}} \int_{0}^{\infty}\left\langle p_{\|}(\beta, t)+p_{\perp}(\beta, t)\right\rangle_{\beta} \mathrm{d} t \\
=k_{\mathrm{fr}}\left\langle\hat{p}_{\|}(\beta)+\hat{p}_{\perp}(\beta)\right\rangle_{\beta}
\end{gathered}
$$

where the symbol \langle\rangle$_{\beta}$ denotes averaging over all orientations and $\hat{p}_{\|}$and $\hat{p}_{\perp}$ denote the Laplace transforms $(s \rightarrow$ 0 ).

After the Laplace transforms of eq 18 and 19 are taken, they may be solved in a similar but much simpler fashion as eq 1 by orthogonal expansion using the Legendre polynomials $L_{i}(\cos \beta)$

$$
\begin{aligned}
& p_{\|}(\beta)=\sum_{i=0,2, \ldots} a_{i} L_{i}(\cos \beta) \\
& p_{\perp}(\beta)=\sum_{i=0,2, \ldots} b_{i} L_{\mathrm{i}}(\cos \beta)
\end{aligned}
$$

Substituting eq 21 into eq $20 \mathrm{~b}$ yields

$$
\phi_{\mathrm{fr}}=k_{\mathrm{fr}} \frac{a_{0}+b_{0}}{2}
$$

since

$$
\left\langle L_{\mathrm{i}}(\cos \beta)\right\rangle_{\beta}=2 \delta_{i 0}
$$

The following relations for the Legendre polynomials are used to reduce eq 18 and 19 to two systems of coupled linear equations which may be truncated at some order $j$

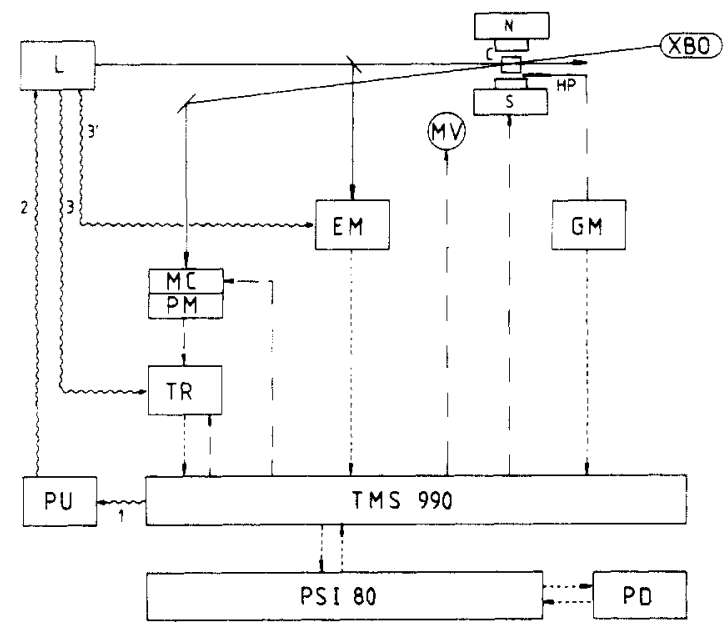

Figure 1. Schematic diagram of the laser flash spectrometer: $C$, cuvette; EM, energy meter; GM, gaussmeter; HP, Hall probe; L, laser; MC, monochromator; NS, electromagnet; PD, peripheral devices; PM, photomultiplier; MV, magnetic valve; PU, pulse unit for the probe light; PSI 80, graphic terminal; TMS 990, microprocessor; TR, transient recorder; ХBO, probe light lamp; - light beams, $\mathrm{m}$, trigger pulse sequence, generated in the order of the numbers; - - - transmission lines for analogue signals; ...., digital data transmission lines.

( $j=8$ is generally sufficient) and solved numerically on a minicomputer.

$$
\begin{gathered}
\nabla_{\beta}{ }^{2} L_{i}=-i(i+1) L_{i} \\
\left\langle L_{i} L_{j}\right\rangle_{\beta}=\delta_{i j} \frac{2}{2 i+1} \\
\left(\cos ^{2} \beta\right) L_{\mathrm{i}}=\frac{(i+1)(i+2)}{(2 i+1)(2 i+3)} L_{i+2}+\frac{2 i^{2}+2 i-1}{(2 i+3)(2 i-1)} L_{i}+ \\
\frac{i(i-1)}{(2 i+1)(2 i-1)} L_{i-2}
\end{gathered}
$$

In concluding this section, we note that the exact result for the zero-field and the high-field case can be obtained without much computational effort. The numerical solution of the full problem requires computer memory of some 300 kbyte with computation times of typically $1 \mathrm{~s}$ on a large computer (IBM 174, CDC 6600) to calculate the radical yield for one set of given parameters.

\section{Experimental Section}

Instrumentation. The magnetic field dependent measurements were performed in a kinetic laser flash spectrometer with a flash lamp pumped dye laser. The electroopitcal setup was basically the same as described previously. ${ }^{13}$ Major improvements were achieved, however, by use of a flash lamp pumped Lambda Physik FL 3000 dye laser, which allows pulse repetition rates up to $20 \mathrm{~s}^{-1}$ (pulse energy about $10 \mathrm{~mJ}$, pulse width $0.8 \mu \mathrm{s}$ ). Thus, very efficient signal averaging became possible in a reasonable measuring time. In order to handle the data acquisition from the transient recorder and the other instruments such as the energy meter and the gaussmeter at a rate compatible with the laser repetition frequency, we found it necessary to put the apparatus under microprocessor control. The different components and their organization in the apparatus are depicted schematically in Figure 1.

The operator communicates with the processor and controls the apparatus via a Kontron Psi 80 microcomputer, used as an intelligent terminal and equipped with a graphic CRT, two floppy disk units, and a matrix line printer. The software of the microprocessor (Texas In-

(13) U. Steiner, Z. Naturforsch. A, 34, 1093 (1979). 
struments TM 990 microcomputer based on the 16-bit 9900 microprocessor chip) permits several modes of operating the spectrometer, e.g., averaging the same decay curve at a fixed wavelength, scanning the monochromator and recording automatically a flash spectrum at several selected delay times after the laser flash, or modulating the magnetic field during a series of laser pulses. We shall briefly describe the latter of these modes, since it is the most relevant in relation to the present work.

Under control of the magnetic field modulation mode, the microprocessor varies the magnetic field strength between selected values of the magnetic field (normally two, one of them zero field) at preset intervals of groups of laser pulses. Each experiment comprises a given number of laser pulses (usually several hundred), whereby each pulse occurs within a repeated cycle of the same operations.

The cycle starts with arming the transient recorder and a trigger signal output from the processor to the probe light pulse unit (home made, providing 1-ms pulses of 4-kW electrical power to a 150-W Osram XBO lamp). The pulse unit, after reaching constant pulsed output, generates a trigger pulse for the laser which in turn triggers the transient recorder (datalab 922, conversion rate up to $20 \mathrm{MHz}$ ) and the energy meter (laser precision $R k 3230$, equipped with a RkP-335 energy probe) for laser pulse energy detection. After the transient recorder has finished recording, the processor accepts the data from the transient recorder, the energy meter, and the gaussmeter (Bell 615 with STG 1-40 Hall probe). Only a selected set of channels of the transient recorder, corresponding to the signal amplitude at certain selected delay times after the laser pulse, is stored by the processor. The cycle is completed by replacing the solution in the cuvette, by opening for a while the magnetic valve in the flow system, and, depending on the program and the progress of the experiment, the magnetic field will be set to a new value or remain unchanged.

Between two cycles the present state of the processed signal is transferred to the Psi 80 microcomputer for storage and display, allowing the operator to observe the measurement at any time.

An example of the diagram produced in such an experiment is shown in Figure 3. By plotting successively the signal amplitude at a certain delay time and, in the same diagram, the corresponding magnetic field settings we obtained an instructive picture of the magnetic field effect in relation to the statistical scatter of the signal amplitude.

Materials and Methods. Sources and purification of the materials used were the same as published in ref 9 . The investigations were performed on methanolic solutions of thionine $\left(3 \times 10^{-5} \mathrm{M}\right)$ and the various halogen-substituted anilines $\left(2 \times 10^{-3} \mathrm{M}\right)$. The solutions were buffered at $\mathrm{pH}$ 8.6 by adding $0.015 \mathrm{M}$ phenylacetic acid and $0.005 \mathrm{M}$ sodium methylate in order to quantitatively protonate the produced dye semiquinone radicals to a protolytic form most convenient for quantitative spectral detection (cf. ref 14).

Before measurement, the solution was flushed with nitrogen for $0.5 \mathrm{~h}$ in order to remove oxygen. After this procedure the solution was transferred to the cuvette (optical path length $10 \mathrm{~mm}$ ) via a teflon pipe (2.5- $\mathrm{mm}$ cross section), the flow being driven by a $0.2 \mathrm{~atm}$ of $\mathrm{N}_{2}$ transport pressure and controlled by the opening time of a magnetic valve, so that the cuvette volume of $0.3 \mathrm{~mL}$ was replaced after each laser pulse. The teflon pipe was contained in

(14) U. Steiner, G. Winter, and H. E. A. Kramer, J. Phys. Chem., 81, 1104 (1977).

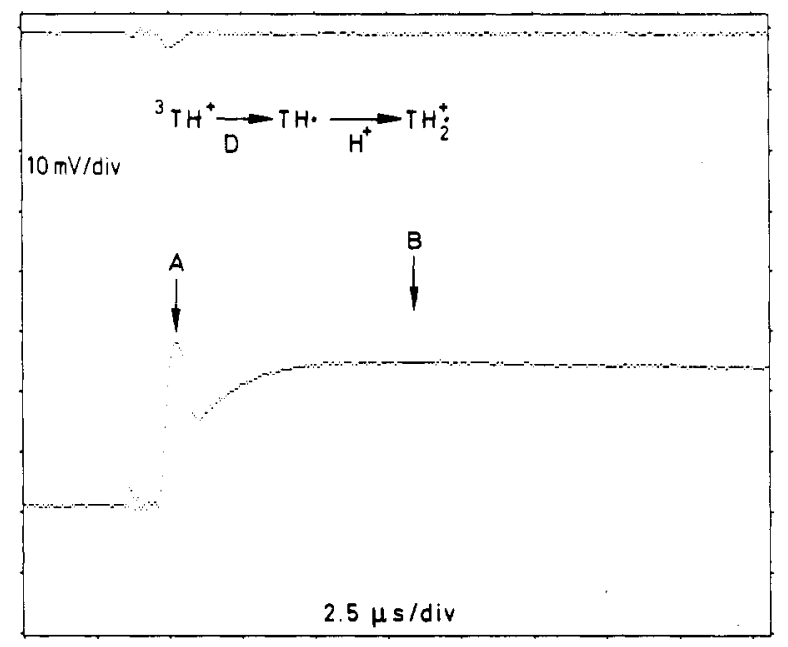

Figure 2. Typical transient observed at $780 \mathrm{~nm}$ when flashing a solution of thionine $\left(3 \times 10^{-5} \mathrm{M}\right)$ with the electron donor (D) $p$-iodoaniline $\left(2 \times 10^{-3} \mathrm{M}\right)$ in methanol butfered at $\mathrm{pH}$ 8.6: upper trace, signal due to stray light; lower trace, transmission of a solution in a $1-\mathrm{cm}$ cuvette. The traces represent averages of 10 signals. A and B mark delay times referred to in Figure 3.

a 10-mm-wide polyethylene pipe flushed with $\mathrm{N}_{2}$ in order to prevent $\mathrm{O}_{2}$ diffusion from the ambient atmosphere, a process which was extremely fast if the teflon pipe was directly exposed to air.

The laser dye used was rhodamine 6G (Lambdachrome 5900 ) and the laser wavelength was tuned to $590 \mathrm{~nm}$, which is close to the first absorption maximum of the dye thionine. In order to keep the observed signal amplitudes proportional to the laser intensity, and hence also to the actual quantum yield of radical formation, we adjusted the laser intensity so as to keep the fraction of the dye, reacting in a single-shot excitation, below $30 \%$.

\section{Results}

A typical transient signal obtained when quenching the thionine triplet ${ }^{3} \mathrm{TH}^{+}$by a halogen-substituted aniline is shown in Figure 2. The signal was recorded at $780 \mathrm{~nm}$, where the triplet and the semithionine radical $\mathrm{TH}_{2}{ }^{+}$. have absorption maxima. ${ }^{14}$ The radical form $\mathrm{TH}$., however, obtained primarily from the triplet by electron transfer, does not absorb at this wavelength. With a quencher concentration of $2 \times 10^{-3} \mathrm{M}$, the actual triplet lifetime is about $0.2 \mu \mathrm{s}$. This is considerably shorter than the duration of the laser pulse $(0.8 \mu \mathrm{s})$ and hence the apparent triplet absorption exhibits the time characteristics of the exciting laser pulse.

The decay of the triplet absorption can be seen only because the radicals $\mathrm{TH}$. formed with some yield in the quenching reaction do not absorb at the particular observation wavelength. After the laser pulse the signal increases again with a time constant of about $2 \mu \mathrm{s}$. This corresponds to the formation of the radical $\mathrm{TH}_{2}{ }^{+}$. by protonation of the radical TH. The lifetime of $\mathrm{TH}_{2}{ }^{+}$. under the present conditions is of the order of some 100 $\mu \mathrm{s}$ so that the decay is not observed on the time scale of Figure 2. The signal amplitude after about $6 \mu \mathrm{s}$ is taken as a direct measure of the yield of radicals formed in the quenching reaction.

Figure 3 shows how an external magnetic field influences the transient signal. The diagram shown was obtained by running the spectrometer in the "magnetic field modulation mode" as described in the Experimental Section. The transient signal of the kind in Figure 2 was repeatedly measured in a series of 350 laser pulses, where for each single pulse the pulse energy, the magnetic field strength, 


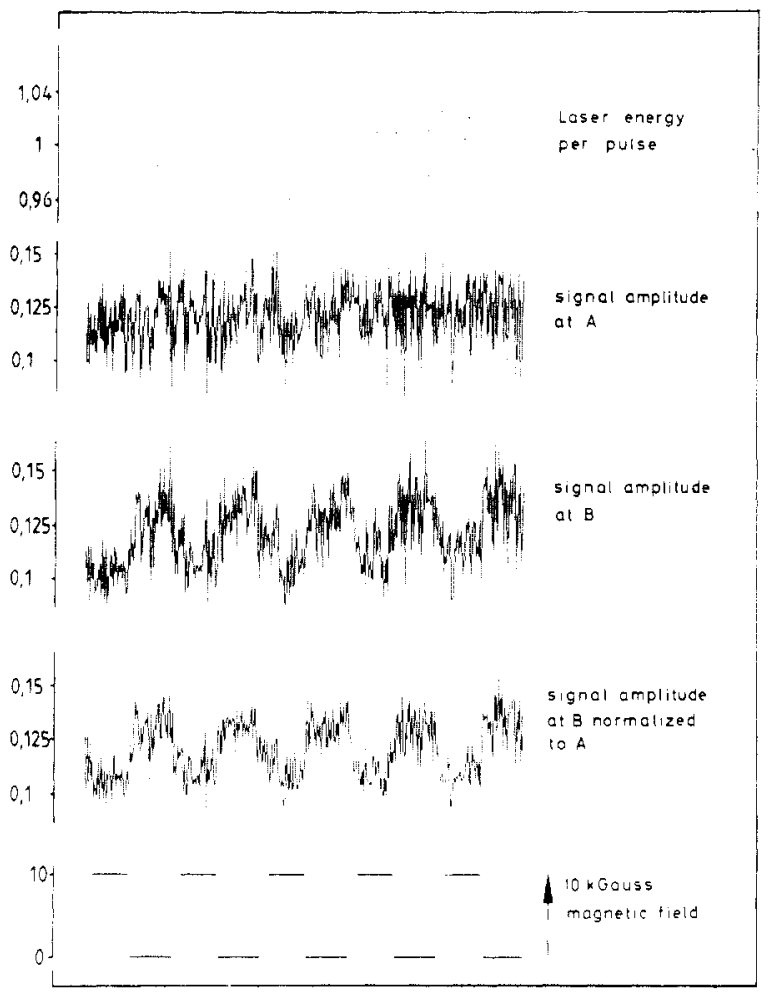

Figure 3. Magnetic field effect on the signal displayed in Figure 2. The results of 350 single-shot experiments are recorded: upper trace, each point represents the energy of a laser pulse (relative units); middle traces, optical densities at delay times A or B, respectively (for clearer representation subsequent data points are connected by straight lines): lower trace, magnetic field.

and the signal amplitude at some delay time (points $\mathrm{A}$ and $B$ in Figure 2) were detected and plotted in succession for each pulse. Thus the three traces in the respective diagrams in Figure 3 were obtained.

In the present example, the magnetic field was switched between zero field and $10 \mathrm{kG}$ after every 35 laser pulses. Figure 3 shows that the signal amplitude at $A$, which is a measure of the amount of triplet formed, is not magnetic field sensitive. The signal amplitude as well as the laser pulse energy show considerable scatter but do not correlate with the magnetic field modulation. On the other hand, as shown in Figure 3, the signal amplitude at B clearly responds to the changes in the magnetic field strength. The signal is lower at high fields.

The signal scatter can be considerably reduced by normalizing the amplitude at $B$, corresponding to the amount of radicals produced, to either the signal amplitude at $A$, where it reflects the amount of triplets which have reacted, or to the laser pulse energy. The former normalization is shown in Figure 3. Normalization to the laser energy improves the signal-to-noise ratio to a similar extent.

The results displayed in Figure 3 unequivocally demonstrate that the magnetic field effect is connected with the radical formation from the triplet and not with the triplet production. Moreover, experiments in unbuffered solution, where the $\mathrm{TH}$ - radical formation was observed at $420 \mathrm{~nm}$, showed that it is really the primary radical formation which is magnetic field sensitive and not the protolytic transformation of $\mathrm{TH}$. into $\mathrm{TH}_{2}{ }^{+}$. As is apparent from Figure 3, where the laser repetition rate was 2 pulses $\mathrm{s}^{-1}$, it takes about $3 \mathrm{~s}$ until the magnetic field rises from zero field to a constant value of $10 \mathrm{kG}$. To evaluate the relative magnetic field effect, we took into account only the data points with the magnetic field fully adjusted. By averaging the magnetic field effect over a trace of the kind

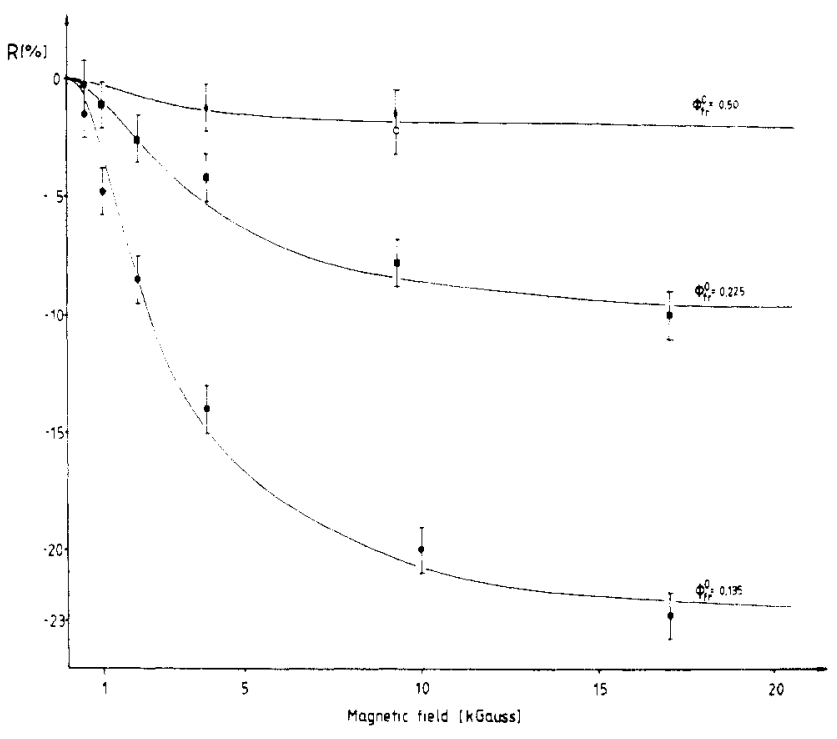

Flgure 4. Relative magnetic field effect $R$ on the radical yield observed on quenching the thionine triplet with various halogen-substituted anilines $\left(2 \times 10^{-3} \mathrm{M}\right)$ in methanol, buffered at $\mathrm{pH}$ 8.6: $p$-iodoaniline; $\square, o$-iodoaniline; $\uparrow, m$-iodoaniline; $\square, p$-bromoaniline. The solid lines were calculated with the parameters given in Table II. The $\phi_{\pi}{ }^{\circ}$ values given are the absolute quantum yields at zero field used for the calculation (cf. ref 19).

shown in Figure 3 we found it possible to obtain a meansquare deviation of about $2.5 \%$, which was further improved to about $1 \%$ by averaging over several traces.

In our experiments, the magnetic field modulation amplitude was varied up to $17 \mathrm{kG}$. The relative magnetic field effects $R$ (cf. eq 27) observed with the iodoanilines and

$$
R=\frac{\phi_{\mathrm{fr}}(B)-\phi_{\mathrm{fr}}^{0}}{\phi_{\mathrm{fr}}^{0}}
$$

$p$-bromoaniline are shown in Figure 4. As noted previously, ${ }^{13}$ the effects observed correlate inversely with the absolute radical yield in the quenching reaction with the respective halogen-substituted aniline. For $m$-iodoaniline and $p$-bromoaniline they are just detectable, but the detailed magnetic field dependence cannot be resolved at the present stage of experimental accuracy.

The solid lines shown in Figure 4 represent the theoretical results obtained by numerical solution of the SLE, where the parameters were determined by a fitting procedure described below.

\section{Discussion}

Before analyzing the observed magnetic field effect in terms of the triplet mechanism, we will give a short consideration to the possible contribution of the radical pair mechanism.

Dissociation of the triplet exciplexes leads to geminate radical pairs with triplet spin alignment. Cage recombination of these pairs regenerating the initial molecules in their singlet ground states is only possible if tripletsinglet transitions have occurred in the radical pairs during the time intervals between probable reencounters. Such isc may be induced by hyperfine interaction, a mechanism which is largely suppressed by increasing the magnetic field strength..$^{15}$ On the other hand, if there is a difference between the $g$ factors of the radicals, this will induce enhanced isc with increasing magnetic field. ${ }^{16}$ This so-called $\Delta g$ mechanism was previously invoked by us ${ }^{13}$ in order to

(15) Cf., e.g., K. Schulten, H. Staerk, A. Weller, H.-J. Werner, and B. Nickel, Z. Phys. Chem. (Frankfurt am Main), 101, 371 (1976).

(16) R. Kaptein, J. Am. Chem. Soc., 94, 6251 (1972). 


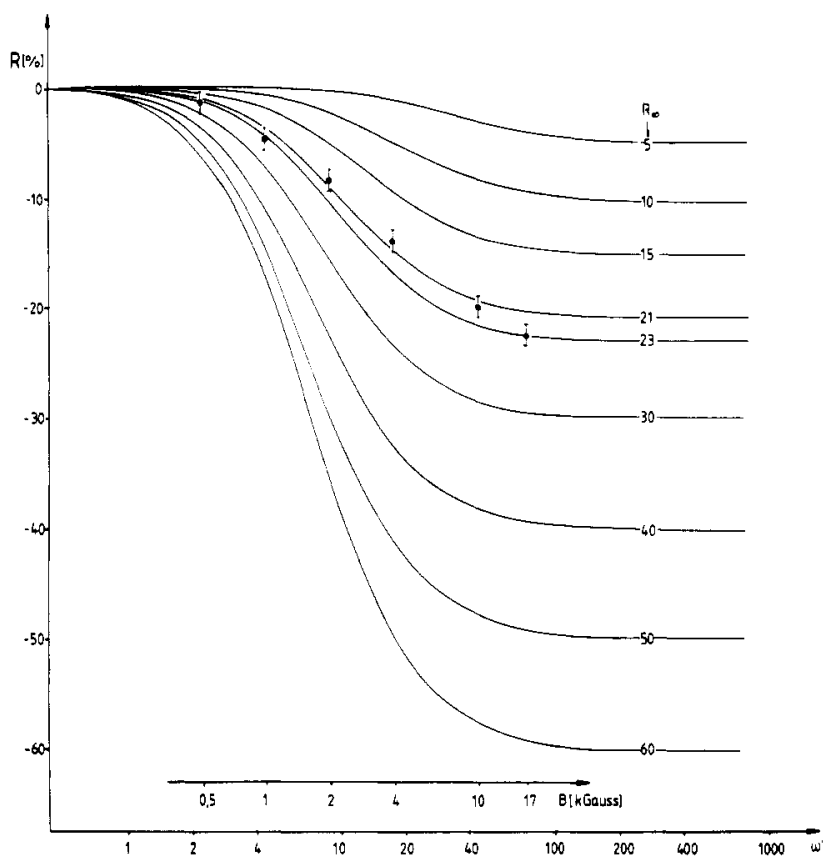

Figure 5. Relative magnetic field effect $R$ calculated as a function of the Larmor frequency $\omega^{\prime}$ (log scale), for $\phi_{*}^{0}=0.135, z=0$, and $D^{\prime}$ $=0$ with various values of $R_{\infty}$. Also plotted are the experimental data points for $p$-lodoanline over a log scale of the magnetic field $B$. The relative position of the $\log B$ scale is adjusted so as to obtain a good fit of theoretical curves with the experimental data points.

explain the heavy atom dependent magnetic field effect in our systems. If we assume, however, for $\Delta g$ a value of 0.01 , which may be a good value as an upper limit for $\pi$-type radicals even if heavy atom substituents are involved, the quantitative theory 17 predicts magnetic field effects of only about $1.5 \%$ at $10 \mathrm{kG}$. Thus the $\Delta g$ effect, even if it is present in our systems, has a maximum contribution to the magnetic field effect which is on the order of magnitude of the error limits in our experiments.

Moreover, the hyperfine mechanism should counteract the $\Delta g$ mechanism. Using the semiclassical treatment of Schulten and Wolynes, ${ }^{18}$ we can estimate as an upper limit for the hyperfine effect a value of $+1 \%$. This is not observed, however, even at low fields where it should dominate the $\Delta g$ mechanism. Thus we conclude that, mainly due to the lack of Coulomb attraction in the radical pairs formed, the contribution of the radical pair mechanism is only of minor importance and may be neglected in the interpretation of the effects described in this paper.

Determination of Kinetic Exciplex Parameters. According to eq 11 the radical yield is determined by the parameters $k_{\mathrm{fr}}, k_{\mathrm{isc}}, z, D_{\mathrm{r}}$, and $D$ and by the Larmor frequency $\omega$. We will now discuss to what extent the parameters can be fixed by the observed magnetic field dependence of the radical yield $\phi_{\mathrm{fr}}$.

At first we note that $\phi_{\mathrm{fr}}$ does not depend on the absolute values of the kinetic and energetic parameters but only on their ratios, which may be expressed as follows:

$$
\phi_{\mathrm{fr}}\left(k_{\mathrm{fr}}, k_{\mathrm{isc}}, z, D_{\mathrm{r}}, D, \omega\right)=\phi_{\mathrm{fr}}\left(k_{\mathrm{isc}}^{\prime}, z, D_{\mathrm{r}}^{\prime}, D^{\prime}, \omega^{\prime}\right)
$$

with

$$
\begin{aligned}
k_{\mathrm{isc}}^{\prime} & =k_{\mathrm{isc}} / k_{\mathrm{fr}} \\
D_{\mathrm{r}}^{\prime} & =D_{\mathrm{r}} / k_{\mathrm{fr}} \\
D^{\prime} & =D / k_{\mathrm{fr}} \\
\omega^{\prime} & =\omega / k_{\mathrm{fr}}
\end{aligned}
$$

(17) K. Schulten and I. R. Epstein, J. Chem. Phys., 71, 309 (1979). (18) K. Schulten and P. Wolynes, J. Chem. Phys., 68, 3292 (1978).
TABLE I: Parameters Used for the Calculation of the Curves Shown in Figure $5^{a}$

\begin{tabular}{ccc}
\hline$R_{\infty}, \%$ & $D_{Y}^{\prime}$ & \multicolumn{1}{c}{$k_{\text {isc }}^{\prime}$} \\
\hline 5 & 7.444 & 10.336 \\
10 & 3.752 & 11.127 \\
20 & 1.892 & 12.974 \\
30 & 1.262 & 15.344 \\
40 & 0.944 & 18.505 \\
50 & 0.752 & 22.936 \\
60 & 0.623 & 29.638 \\
90 & 0.405 & 134.939 \\
${ }^{a} \phi_{\mathrm{fr}^{\circ}}{ }^{\circ}=0.135, z=0, D^{\prime}=0$. &
\end{tabular}

In Figure 5 a family of curves characterizing the magnetic field effect is shown. The curves were obtained from the numerical solution of the SLE as described above. We use a $\log$ scale for the Larmor frequency $\omega^{\prime}$ so that a direct comparison of the respective curve shape with experimental results is possible even though $k_{\mathrm{fr}}$ (cf. eq $29 \mathrm{~d}$ ) is not known. The curves are calculated with $D^{\prime}$ and $z$ equal to zero, their influence being considered below, and with a zero-field radical yield $\phi_{\mathrm{fr}}^{0}$ of 0.135 , corresponding to the experimental result for the $p$-iodoaniline system. Fixing $\phi_{\text {fr }}^{0}$ and the magnetic field effect at infinite field $R_{\infty}$ uniquely defines the parameters $k_{\mathrm{isc}}^{\prime}$ and $D_{\mathrm{r}}^{\prime}$ by means of eq 13 and 20 . The respective values are given in Table $I$.

From Table I we see that the high-field magnetic field effect $R_{\infty}$ increases monotonically with decreasing $D_{\mathrm{r}}^{\prime}$, which is to be expected since, qualitatively, the effect of the magnetic field is equivalent to the effect of rotational diffusion. Thus, if $D_{x}^{\prime}$ is large, there are already fast transitions among the triplet sublevels at zero field and the magnetic field cannot add much to the effect, whereas the opposite is true if $D_{r}^{\prime}$ is small.

The curves shown in Figure 5 have a typical S-shaped form, symmetric with respect to their inflection point. This inflection point, where the magnetic field effect reaches half of its maximum value $R_{\infty}$ at high fields, occurs at Larmor frequencies $\omega_{1 / 2}^{\prime}$ obeying the condition $\omega_{1 / 2}^{\prime} \approx$ $6 D_{r}^{\prime}$ if $6 D_{r}^{\prime}>1$, which again emphasizes the intimate relation between molecular rotation and the Larmor precession.

In Figure 5 we have also plotted our experimental data for the $p$-iodoaniline system as a function of $\log B$ with an arbitrary shift of the abscissa, so that an optimum fit with theoretical curves is obtained. As can be seen, the experimental data can only be fitted to the theoretical curves with $R_{\infty}=21-23 \%$. If we adopt the curve with $R_{\infty}$ $=21 \%$, the data point at $17 \mathrm{kG}$ is clearly off by $2 \%$, whereas with $R_{\infty}=23 \%$ there is a relatively strong deviation of the data point at $1 \mathrm{kG}$. As will be shown below, even additional allowance for nonzero values of $z$ and $D^{\prime}$ cannot account for this basic shortcoming in the fit. Therefore one may suspect that, although the theory of the triplet mechanism developed so far accounts fairly well for the observed magnetic field dependence, there is some residual effect which awaits further clarification. For further evaluation of the $p$-iodoaniline exciplex parameters, we shall prefer to use $R_{\infty}=23 \%$, giving better account of the high-field values. However, use of $R_{\infty}=21 \%$ would not significantly change the parameters which are finally obtained.

We shall now turn to the role of the zfs-parameter $D$ (or $D^{\prime}$ in dimensionless units) and the parameter $z$ defining the degree of spin selectivity in the isc process. We note that in the zero-field and high-field cases $\phi_{\mathrm{fr}}$ is independent of $D^{\prime}$. Hence we may begin by establishing the restrictions imposed on $k_{\mathrm{isc}}^{\prime}, D_{\mathrm{r}}^{\prime}$, and $z$ by the quantum yield $\phi_{\mathrm{fr}}^{0} \mathrm{ob}-$ served at the zero-field and by the high-field limit $R_{\infty}$ of 


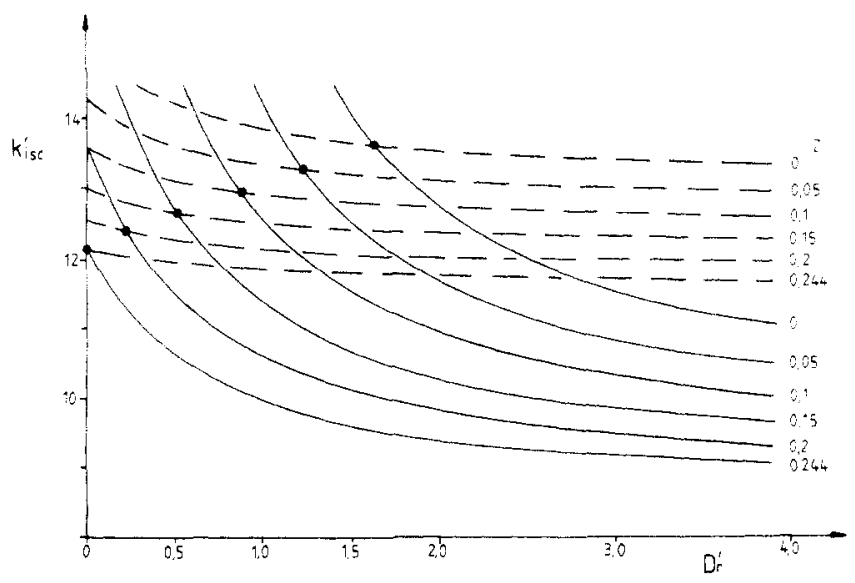

Figure 6. Parameter variation compatible with fixed values for the zero-field quantum yield $\phi_{f r}{ }^{\circ}=0.135$ (solid lines) and the high-field quantum yield corresponding to $R_{\infty}=0.23$ (dashed lines). The intersection points where zero-field and high-field conditions are fulfilled simultaneously are indicated.

the effect adopted from Figure 5.

Fixing some parametric value for $z$ defines a functional relation between $k_{\text {isc }}^{\prime}$ and $D_{\mathrm{r}}^{\prime}$ for both the zero-field case (using eq 13) and the high-field case (using eq 22). These functions are graphically represented by the curves in Figure 6 for $p$-iodoaniline.

In the zero-field case, the influence of $D_{\mathrm{r}}^{\prime}$ is much more pronounced (the curves are steeper) than in the high-field case. This is a consequence of the fact that, at high fields, most of the transitions occurring between the triplet substates in the molecular frame are induced by the Zeeman interaction, whereas at zero field they are only due to molecular rotational diffusion, the time constant of which is directly proportional to $D_{r}^{\prime}$. The intersection points of the corresponding zero-field and high-field curves uniquely determine $k_{\text {isc }}^{\prime}$ and $D_{\mathrm{r}}^{\prime}$ for a particular $z$.

It is important to note from Figure 6 that the zero-field and high-field conditions can be met simultaneously only if $z \leq 0.244$, which means that the degree of sublevel selectivity in the isc process deactivating the triplet exciplex must be fairly pronounced.

After determining $k_{\text {isc }}^{\prime}$ and $D_{r}^{\prime}$ for particular values of $z$, the full numerical treatment may be applied to calculate $\phi_{\mathrm{fr}}$ as a function of $\omega^{\prime}$. The choice of $D^{\prime}$ will leave unaffected $\phi_{\mathrm{fr}}(\omega=0)$ and $\phi_{\mathrm{fr}}(\omega \rightarrow \infty)$ but it will influence the magnetic field dependence of the radical yield at intermediate fields, so that formation on $D^{\prime}$ can be extracted from the particular shape of the magnetic field effect curve.

In order to explicitly compare the calculated magnetic field effect, obtained as a function of $\omega^{\prime}$, with the measured effect, obtained as a function of $\omega$, we have to know $k_{\mathrm{fr}}$. This would also fix our absolute values of $k_{\text {isc }}, D_{\mathrm{r}}$, and $D$. Since changing the abscissa from $\omega$ to $\omega^{\prime}=\omega / k_{\mathrm{fr}}$ merely corresponds to a linear expansion or contraction of the abscissa, one may use a logarithmic scale for $\omega$ and $\omega^{\prime}$. This will horizontally displace the curves $\phi_{\mathrm{fr}}$ vs. $\log \omega$ by $-\log$ $k_{\text {fr }}$ when going from the $\log \omega$ to the $\log \omega^{\prime}$ scale, but the curve shape will remain unchanged.

From these considerations we derive the following procedure to find the best fit for $D^{\prime}$ and $k_{\text {fr. }}$ Keeping $k_{\text {isc }}^{\prime}$ and $D_{\mathrm{r}}^{\prime}$ fixed as determined by the value of $z$, we calculate a set of curves $\phi_{\mathrm{f}, \text { calod }}\left(\log \omega^{\prime}\right)$ with $D^{\prime}$ as a running parameter. Then we compare the experimentally determined values $\phi_{\mathrm{ff}, \text { obed }}$, plotted vs. $\log \omega_{\text {expt }}$, with the set of curves $\phi_{\mathrm{fr}, \text { caled }}(\log$ $\left.\omega^{\prime}\right)$ and find the best fit in curve shape, which in turn yields $D^{\prime}$. The required shift to bring $\phi_{\text {fr,calcd }}$ and $\phi_{\text {fr,obed }}$ into best coincidence is equal to $\log k_{\mathrm{fr}}$. Thus $k_{\mathrm{fr}}, k_{\mathrm{isc}}, D_{\mathrm{r}}$, and $D$ can be obtained as functions of $z$.
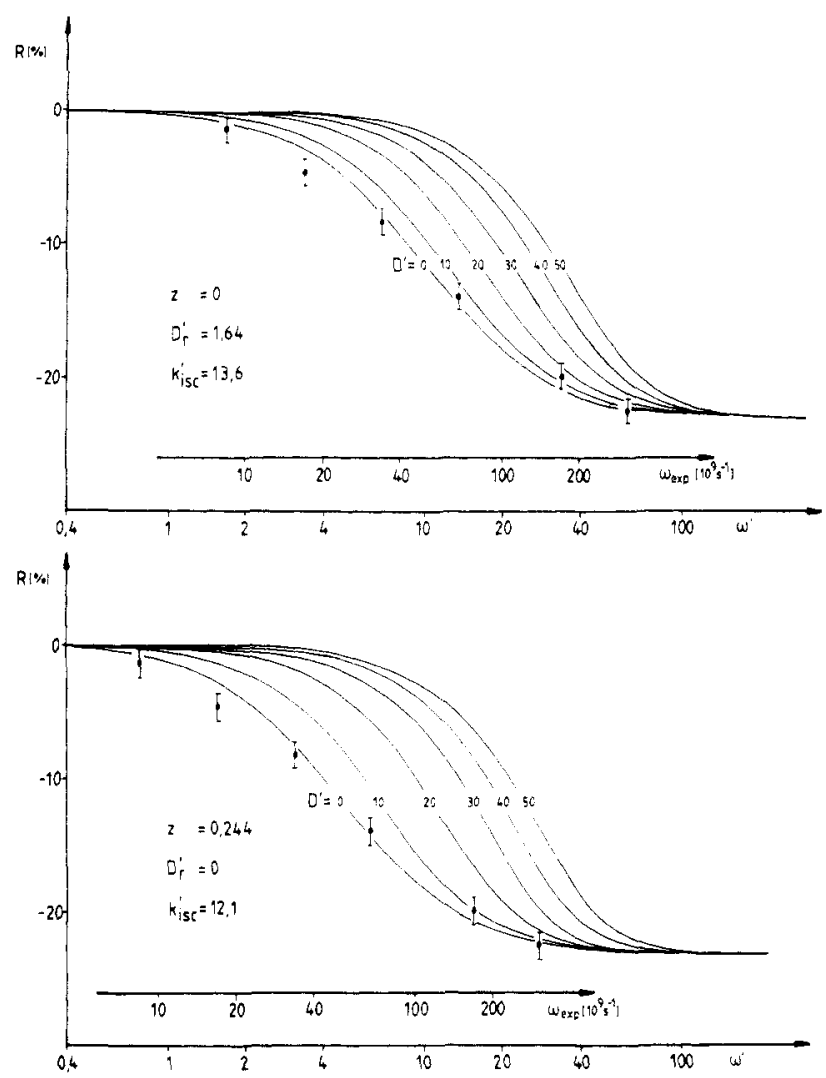

Figure 7. Influence of $z$ parameter and $z$ fs parameter $D^{\prime}$ on the magnetic field dependence of the relative magnetic field effect $R$ for $\phi_{\pi}{ }^{0}=0.135$. $R$ is plotted vs. $\log \omega^{\prime}$; the experimental points for the $p$-iodoaniline system are plotted vs. $\log \omega_{\text {expt }}$ (actual Larmor frequency), the horizontal displacement being chosen so as to give a best fit with the $D^{\prime}=0$ curve.

The procedure is demonstrated in Figure 7 for $p$-iodoaniline in the cases where $z$ adopts its smallest and largest possible value. For each $z$ the family of curves with a set of equidistant $D^{\prime}$ values is shown. On increasing $z$ the major effect is a horizontal displacement of the curves to lower $\omega^{\prime}$ on the $\log \omega^{\prime}$ scale. Furthermore, the spread of the curve family becomes wider with increasing $z$. For each $z$ the best fit with the experimental points is achieved if $D^{\prime}=0$. However, if we accept mean-square deviations corresponding to the experimental ones, the actual $D^{\prime}$ parameter could be in the range between 0 and 10 .

Since for $D^{\prime}=0$ the shape of the curves is independent of $z$, this is also true for the accuracy of the best fit. Thus the allowed range of $z$ values is not further narrowed by the fitting procedure. The parameters $k_{\mathrm{fr}}, k_{\mathrm{isc}}$, and $D_{\mathrm{r}}$ obtained as functions of $z$ in its allowed range are plotted in Figure 8 for the $p$-iodoaniline exciplex.

So far our reasoning has been purely mathematical and we have not used criteria of physical significance for the parameters in order to narrow the possible ranges for their values. Focussing attention on the physical origin of $D_{\mathrm{r}}$ will, however, reduce the "free range" of $z$ and the other parameters.

We note that $D_{\mathrm{r}}$ has been introduced as the isotropic rotational diffusion constant of the exciplex. When Debye's theory ${ }^{20}$ is used, it can be related to solvent viscosity

(19) H.-P. Waschi and U. Steiner, to be published. The following values of the radical yields slightly correct the previous results ${ }^{9}$ given in parentheses: $p$-iodoaniline, $0.135(0.11)$; $o$-iodoaniline, $0.225(0.21) ; \mathrm{m}$ iodoaniline, 0.45 (0.46); $p$-bromoaniline, $0.51(0.48)$.

(20) P. Debye, “Polar Molecules”, Dover Publications, New York, 1945. 


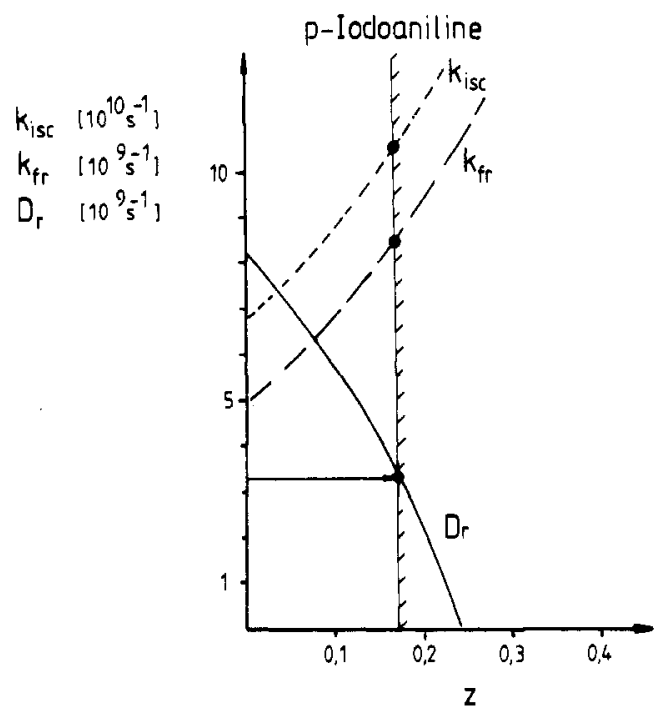

Figure 8. Parameters $k_{\text {bec }}, k_{r}$, and $D_{r}$ as functions of $z$. The corresponding parameter sets give equally good fits of the theoretical with the experimental results for $p$-lodoaniline. The upper bound of $z$ is obtained from the lower bound of $D_{\mathrm{r}}$ (cf. text).

$\eta$, absolute temperature $T$, and the effective hydrodynamic radius $r$ of the triplet exciplex by

$$
D_{\mathrm{r}}=\frac{k T}{8 \pi \eta r^{3}}
$$

Although the exact exciplex geometry is not known, an upper limit for the effective hydrodynamic exciplex radius may be estimated. As demonstrated in the previous theoretical treatment ${ }^{8 b}$ of the isc deactivation of the triplet exciplex, spin-orbit coupling is expected to be sensitive to the spin orientation relative to the aniline moiety, incorporating the heavy atom and determining the local symmetry of the heavy atom substituent. Thus $D_{\mathrm{r}}$ will actually refer to the reorientation of this exciplex component. If we assume a fairly compact sandwich configuration, which would be attractive in the sense that it allows one to invoke transannular orbital interaction for the exciplex bonding, $D_{\mathrm{r}}$ would be determined by the motion of the exciplex as a whole.

If other types of weak bonding, e.g., hydrogen bonds between the exciplex components, were considered this would give the components a higher orientational mobility relative to each other than in the sandwich structure. Therefore the rigid sandwich configuration, giving us a higher value for the effective hydrodynamic radius $r$, may safely be used to estimate an upper bound for this quantity.

In the sandwich configuration the exciplex could be packed into the volume of a box with side lengths 12,5 , and $6 \AA$ (cf. Figure 9), with a resultant effective radius of $4.4 \AA$. Adopting the condition $r \leq 4.4 \AA$, i.e. $D_{r} \geq 3.3 \times$ $10^{9} \mathrm{~s}^{-1}$, narrows the range of possible parameters compatible with the experimental results.

In Figure 8 the free range of possible parameters for the $p$-iodoaniline exciplex case is shown. Assuming ideal spin selectivity (i.e., $z=0$ ), we obtain $D_{\mathrm{r}}=8.2 \times 10^{9} \mathrm{~s}^{-1}, k_{f \mathrm{r}}=$ $5 \times 10^{9} \mathrm{~s}^{-1}$, and $k_{\text {isc }}=6.8 \times 10^{10} \mathrm{~s}^{-1}$. On the other hand, for the minimum conceivable value of $D_{\mathrm{r}}$ estimated as 3.3 $\times 10^{9} \mathrm{~s}^{-1}$ we obtain $z=0.175, k_{\mathrm{fr}}=8.5 \times 10^{9} \mathrm{~s}^{-1}$, and $k_{\text {isc }}$ $=10.6 \times 10^{10} \mathrm{~s}^{-1}$. This means that the spin selectivity must in any case be high, and that the decay constants of the triplet exciplex are defined within rather narrow ranges of $\pm 25 \%$.

With the value of $k_{\mathrm{fr}}$ known, the values for $D^{\prime}$ may be converted to $D$ yielding a range of 0 to about $2 \mathrm{~cm}^{-1}$.

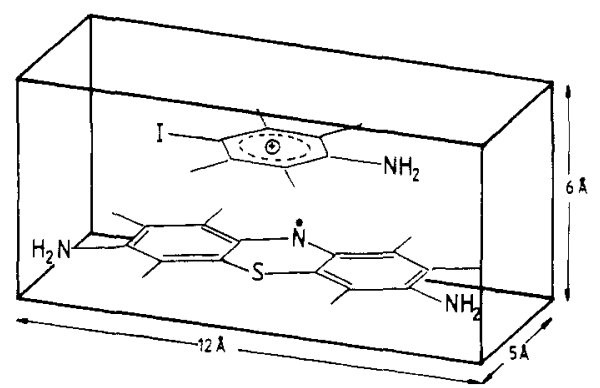

Figure 9. Tentative exclplex geometry used to estimate an upper bound to the effective hydrodynamic radius.

Usually zfs parameters of organic compounds are smaller than $0.2 \mathrm{~cm}^{-1}$ and for exciplexes still smaller values are expected. ${ }^{21,22}$ Thus we see that the chemical kinetic effect of the triplet mechanism in low viscous solvents as analyzed in this paper does not yield much information on the zfs parameters. This should be seen as a principal difference to the CIDEP triplet mechanism, where the effect is very sensitive to the value of $D$.

For 0 -iodoaniline we obtain a somewhat larger range of allowed $z$ values (up to 0.3). For $z=0$ we evaluated $D_{\mathrm{r}}$ $=12 \times 10^{9} \mathrm{~s}^{-1}, k_{\mathrm{fr}}=6.9 \times 10^{9} \mathrm{~s}^{-1}$, and $k_{\mathrm{isc}}=4.2 \times 10^{10} \mathrm{~s}^{-1}$. Thus, whereas the dissociation of the $o$-iodoaniline exciplex appears to be somewhat faster than in the $p$-iodoaniline case and the same applies for the orientational relaxation, the isc rate constant is in this case smaller, which has previously been interpreted by the position dependence of the heavy atom effect.

The magnetic field effects for $m$-iodoaniline and $p$ bromoaniline were too small to resolve the magnetic field dependence with sufficient accuracy. The solid curve shown in Figure 4 for this case was calculated by using $z$ $=0, k_{\mathrm{fr}}=6 \times 10^{9} \mathrm{~s}^{-1}, k_{\mathrm{isc}}=9.4 \times 10^{9} \mathrm{~s}^{-1}, D_{\mathrm{r}}=10 \times 10^{9} \mathrm{~s}^{-1}$, and $D=0$, i.e., with values corresponding to the average of the other cases, except for $k_{\text {isc }}$ which was chosen in order to yield a zero-field quantum yield of 0.5 radicals, which was approximately obtained for both of these donors..$^{9,19}$ It is seen that the magnetic field effect observed is of the expected order of magnitude, supporting the assumption of similar structure and bonding of the various halogensubstituted aniline complexes with the thionine triplet. Thus it may be accepted as a general result of the present investigation that the dissociation rate constant of these species is of the order of $5 \times 10^{9} \mathrm{~s}^{-1}$.

So far most data on triplet exciplexes have been obtained by phosphorescence measurements in rigid solutions at low temperature ${ }^{23}$ and only few examples of triplet exciplex studies in liquid solution are known. Fairly long-lived triplet exciplexes with prophyrins have been observed in nonpolar solvents at room temperature by Whitten and co-workers ${ }^{24}$ and by Kapinus et al. ${ }^{25}$ These exciplexes are spectroscopically similar to the locally excited triplets and do not show ionic dissociation. For the pyrromellitic dianhydride-mesitylene triplet charge transfer complex an ionic dissociation has been reported by Potashnik et al. ${ }^{26}$ The rate constant observed at -156

(21) H. Beens, J. de Jong, and A. Weller, "Colloque Ampère 15", P Averbuch, Ed., North-Holland, Amsterdam, 1969, pp 289-92.

(22) C. P. Keijzers and D. Haarer, J. Chem. Phys., 67, 925 (1977).

(23) Cf., e.g., the reviews by (a) A. Weller in "The Exciplex", M. Gordon and W. Ware, Ed., Academic Press, New York, 1975, p 23; (b) S. Nagakura in "Excited States", Vol. 2, E. C. Lim, Ed., Academic Press, New York, 1975 , p 321.

(24) D. G. Whitten, J. K. Roy, and F. A. Carroll in ref 23a, p 247; J. K. Roy, F. A. Carroll, and D. G. Whitten, J. Am. Chem. Soc., 96, 6349 (1974).

(25) E. I. Kapinus, V. P. Staryi, and I. I. Dilung, Dokl. Akad. Nauk SSSR, 261, 907 (1981). 
${ }^{\circ} \mathrm{C}$ was about $10^{3} \mathrm{~s}^{-1}$.

Even for singlet exciplexes, information on the rate constant of dissociation to radicals is scarce, since its determination requires not only exciplex fluorescence lifetime measurements but also yield measurements of the dissociation products. For radical ion formation in excited singlet-state electron transfer reactions, it is normally difficult to distinguish between radicals formed via dissociation of an intermediate exciplex and radicals formed directly in the electron transfer process. With magnetic field effects, however, such a discrimination is possible. From a combined evaluation of fluorescence decay, absorption measurements, and magnetic field effects, Weller and co-workers ${ }^{27,28}$ have obtained data on ionic dissociation rate constants of singlet exciplexes between pyrene and dimethylaniline. The data for different solvents could be described by the empirical equation ${ }^{27}$

$$
k_{\mathrm{dis}}^{\operatorname{exc}}=\frac{2.3 \times 10^{9}}{\eta} \exp \left[-\frac{e_{0}^{2}}{\epsilon k T}\left(\frac{1}{d}-\frac{1}{a}\right)\right] \mathrm{s}^{-1}
$$

where $\eta$ is the solvent viscosity in $\mathrm{cP}, d$ the interplanar distance of the exciplex components, and $a$ their respective distance after dissociating to a solvent-shared radical pair. The parameters $e_{0}, \epsilon, k$, and $T$ are the electronic charge of the electron, the dielectric constant of the solvent, Boltzmann's constant, and the absolute temperature. For systems lacking Coulomb attraction as in our cases where one radical is neutral, the exponential term becomes 1 , and for methanol $\left(\eta=0.59 \mathrm{cP}\right.$ at $\left.25^{\circ} \mathrm{C}\right)$ a rate constant of 4 $\times 10^{9} \mathrm{~s}^{-1}$ would be obtained. This value compares surprisingly well with the result obtained for our triplet exciplexes and in fact confirms that singlet and triplet exciplexes with dominant charge transfer structure behave rather similarly in polar solvents.

Contrary to the rate constant $k_{\mathrm{fr}}$, the rate constant $k_{\mathrm{isc}}$ in our systems is determined by the heavy atom effect. For the triplet exciplex with unsubstituted aniline as a donor it is possible to estimate an upper limit for $k_{\text {isc }}$ from the absolute radical yield, ${ }^{19}$ using the assumption that $k_{\mathrm{fr}}$ is the same as for the halogen-substituted anilines. Thus a value of $\leq 10^{8} \mathrm{~s}^{-1}$ is obtained. This result can be compared with rate constants for isc processes converting charge transfer states of singlet exciplexes to their locally excited triplet states. For the pyrene-dimethylaniline singlet exciplex a value of $7 \times 10^{6} \mathrm{~s}^{-1}$ has been reported by Werner et al. ${ }^{28}$ In a recent picosecond laser photolysis study, Okada et al. ${ }^{29}$ determined $k_{\text {isc }}$ for a number of singlet exciplexes consisting of covalently linked pyrene and anilines. The rate constants range between $10^{6}$ and $\geq 2.5 \times 10^{10} \mathrm{~s}^{-1}$,

(26) R. Potashnik, C. R. Goldschmidt, and M. Ottolenghi, J. Phys. Chem., 73, 3170 (1969).

(27) A. Weller, Z. Physik. Chem. (Frankfurt am Main), 130, 129 (1982).

(28) H.-J. Werner, H. Staerk, and A. Weller, J. Chem. Phys., 68, 2419 (1978).

(29) T. Okada, I. Karaki, E. Matsuzawa, N. Mataga, Y. Sakata, and S. Misumi, J. Phys. Chem., 85, 3957 (1981).

(30) The influence of sublevel selective deactivation on triplet spin polarization has been treated, however, for triplets free of spin relaxation in molecular crystals at very low temperature by G. T. Evans, Chem. Phys. Lett., 36, 46 (1975).
TABLE II: Characteristic Parameters of Triplet Exciplexes between Thionine and Halogen-Substituted Anilines Evaluated from the Magnetic Field Effect according to the Triplet Mechanism

\begin{tabular}{|c|c|c|c|c|}
\hline electron donor & $z$ & $k_{\text {isc }}{ }^{a}$ & $k_{\mathrm{fr}}{ }^{a}$ & $D_{\mathrm{r}}^{a}$ \\
\hline $\begin{array}{l}p \text {-iodoaniline } \\
o \text {-iodoaniline } \\
m \text {-iodoaniline, } \\
p \text {-bromoaniline }\end{array}$ & $\begin{array}{l}<0.18 \\
<0.3\end{array}$ & $\begin{array}{l}6.8 \times 10^{10} \\
4.2 \times 10^{10} \\
9.4 \times 10^{9}\end{array}$ & $\begin{array}{l}5.0 \times 10^{9} \\
6.9 \times 10^{9} \\
6 \times 10^{9}\end{array}$ & $\begin{array}{r}8.2 \times 10^{9} \\
1.2 \times 10^{10} \\
10^{10}\end{array}$ \\
\hline $\begin{array}{l}\quad a \text { Values in } s^{-1} \\
D=0 \text { have been } u \\
\text { shown in Figure } 4 \\
\text { values. }\end{array}$ & lese & ameters & ether with & $\begin{array}{l}z=0 \text { and } \\
\text { curves } \\
\text { ce of these }\end{array}$ \\
\hline
\end{tabular}

depending on the solvent and relative mobility of the exciplex components. In view of the additional heavy atom effect in the triplet exciplexes considered in this work it seems understandable that even faster isc may occur in these species.

\section{Conclusions}

The analysis of the magnetic field effect as presented above shows that rather definite conclusions can be drawn concerning the absolute rate parameters of the triplet exciplexes studied in this work. The data are collected in Table II. The heavy atom substituents mainly affect the isc rate constant and, to a lesser degree, the other parameters of the triplet exciplexes. Thus, since the magnetic field effect increases with increasing $k_{\text {isc }}$ and allows one to make a more accurate analysis, the heavy atom substitutent enhanced magnetic field effect provides an excellent means of probing the triplet exciplex dynamics.

The essential information obtained concerning the type of triplet exciplexes studied here is that their dissociation rate constant (with respect to formation of free radicals, completing the charge separation initiated in the electron transfer reaction by which the exciplexes are formed) is approximately $5 \times 10^{9} \mathrm{~s}^{-1}$. This dissociation is only about 10 times slower than the orientational relaxation of the exciplexes $\left(6 D_{\mathrm{r}} \approx 5 \times 10^{10} \mathrm{~s}^{-1}\right)$.

In fact it would be difficult to measure the exciplex dissociation by a method which observes the exciplex directly. To understand this, one should bear in mind that the decay of these exciplexes (even if $k_{\text {isc }}$ is negligible) is faster than the intramolecular $S_{1}-T_{1}$ isc process in the parent dye molecule $\left(k_{\mathrm{S}_{1}-\mathrm{T}_{1}}=2.8 \times 10^{9} \mathrm{~s}^{-1}\right) .{ }^{14}$ Therefore, even if the dye singlet were produced within picoseconds, formation of the triplet and subsequently of the triplet exciplex would be slower than the exciplex decay, so that its decay would not be readily resolvable.

Acknowledgment. We are grateful to Dipl. Chem. H.-P. Waschi for his cooperation with the laser spectrometer and for developing the Psi-80 software. Thanks are also due to Dr. P. Pattison for editing the English of the manuscript. Financial support by the Deutsche Forschungsgemeinschaft and the Fonds der Chemischen Industrie is gratefully acknowledged.

Registry No. Thionine, 581-64-6; $p$-iodoaniline, 540-37-4; o-iodoaniline, 615-43-0; $m$-iodoaniline, 626-01-7; $p$-bromoaniline, 106-40-1. 